\title{
An Upgraded Dual-Band Digital Predistorter Model for Power Amplifiers Linearization
}

\author{
Abraham Pérez-Hernández, Juan A. Becerra, Senior Member, IEEE, \\ María J. Madero-Ayora, Senior Member, IEEE, and Carlos Crespo-Cadenas, Senior Member, IEEE
}

\begin{abstract}
Digital predistortion (DPD) based on Volterra models is commonly employed to counteract nonlinear distortion of power amplifiers. However, when concurrent dual-band signals are transmitted, two-dimensional DPD models are required. In this work an upgrading of a standard dual-band model is proposed and justified using multinomial theorem. The linearization performance of the current proposal has been compared to the unextended model. 5G New Radio signals have been generated to compose a dual-band signal, which later was employed as input signal at Chalmers University of Technology's RF WebLab. Using coefficient selection techniques, the most relevant regressors are shown and the importance of the new extension is proven. Linearization results highlight the benefits of this proposal.
\end{abstract}

Index Terms-Digital predistortion, dual-band signal, power amplifiers, Volterra series.

\section{INTRODUCTION}

D IGITAL predistortion (DPD) techniques have been successfully applied to the linearization of single-band power amplifiers (PAs). However, when more bands are included, as in fifth generation $(5 \mathrm{G})$ of mobile communication systems, the DPD becomes technically impractical since the need for oversampling with respect to the occupied bandwidth exceeds the limit in computational speed of the digital signal processing controller. DPDs are commonly supported by Volterra models, which represent a nonlinear input/output relation through a linear combination of regressors. These are built as multiplications of delayed and conjugated versions of the complex envelope of the input signal.

In literature, concurrent dual-band models divide the modulation bandwidth in two bands and the two-dimensional memory polynomial (MP) Volterra model is used [1]-[3]. Some authors propose a modification in the envelope part of the previous model using sums of both signals' envelope [4], [5], while others implement two-dimensional look-up tables (LUT) to model the crossband distortion [6]. Also, memory effects have been characterized by applying the theory of leading and lagging terms [7], [8].

The authors would like to thank the team at Chalmers University of Technology for providing the web-accessible laboratory that was used in this work.

This research was funded by Ministerio de Economía, Industria y Competitividad (MINECO) of the Government of Spain, grant number TEC201782807-P, and by the European Regional Development Fund (ERDF) of the European Commission.

The authors are with the Departamento de Teoría de la Señal y Comunicaciones, Escuela Técnica Superior de Ingeniería, Universidad de Sevilla, 41092 Seville, Spain (e-mail: abperez@us.es; jabecerra@us.es; mjmadero.es; ccrespo@us.es).
It is widely known that the number of coefficients in Volterra series and the aforementioned models grows exponentially with model order and memory depth. The growth in number of coefficients can be faced by using coefficient selection methods, that select the most relevant coefficients within the regressor pool. Amongst these techniques, the doublyorthogonal matching pursuit (DOMP) [9] algorithm returns a regressor list sorted by their importance and has previously proven superior sorting features, assuring the orthogonality between selected regressors.

This communication proposes an extension for a standard dual-band model, which will be called two-dimensional generalized memory polynomial (2-D GMP) model. The performance of the upgraded model has been proven by coefficient selection in the linearization of a concurrent dual-band PA working with 5G New Radio (5G-NR) signals. This work is organized as follows. First, Section II presents the framework and notation conventions. Next, Section III introduces the upgraded model (2-D U-GMP) that is experimentally validated in Section IV. Section V concludes the work.

\section{Dual-Band Behavioral Models}

Before introducing dual-band models, one of the most widely-used single-band models is reviewed in this Section. Known as generalized memory polynomial (GMP) [10], its expression in a discrete-time form can be written as

$u^{(\mathrm{GMP})}(k)=\sum_{p=1}^{P} \sum_{q_{1}=0}^{Q_{1}} \sum_{q_{2}=-Q_{2}}^{Q_{2}} b_{p, q_{1}, q_{2}} x\left(k-q_{1}\right)\left|x\left(k-q_{1}-q_{2}\right)\right|^{p-1}$,

where $u(k)$ denotes the predistorted signal, $x(k)$ is the input signal, $k$ is the discrete-time index, $P$ is the nonlinear order and $Q_{1}$ and $Q_{2}$ represent the memory depths for the different branches. Although it is expressed as one single equation, in (24) of [10] it is divided into three branches so a physical meaning can be given to each of them. The first one, branch A, with $q_{2}=0$, corresponds to the classic MP model, which imposes the same delay for both the signal $x(k)$ and its envelope. The second one, branch $\mathrm{B}$, with positive values of $q_{2}$, matches with the regressors with a lagging envelope, and branch $\mathrm{C}$, with negative values of $q_{2}$, represents leadingenvelope regressors.

However, when a dual-band signal is treated, single-band models do not perform as well as expected. Therefore a dependency on both separated input signals needs to be added. 
The input/output relation of a dual-band baseband Volterra model follows

$$
\begin{aligned}
& u_{1}(k)=\sum_{j} h_{j, 1} \phi_{j, 1}\left\{x_{1}(k), x_{2}(k)\right\}, \\
& u_{2}(k)=\sum_{j} h_{j, 2} \phi_{j, 2}\left\{x_{1}(k), x_{2}(k)\right\},
\end{aligned}
$$

where $\phi_{j, 1}$ and $\phi_{j, 2}$ are the corresponding regressor generation functions for each case and $j$ sweeps the regressor pool that is model dependent. Each regressor is weighted differently by its Volterra coefficient, $h_{j, 1}$ for the lower band and $h_{j, 2}$ for the upper band. According to literature [1]-[8], [11]-[14], the basic regressor of a dual-band model is

$\phi_{j, i}\left\{x_{1}(k), x_{2}(k)\right\}=x_{i}\left(k-q_{0}\right)\left|x_{1}\left(k-q_{1}\right)\right|^{p_{1}}\left|x_{2}\left(k-q_{2}\right)\right|^{p_{2}}$,

where different configurations of delays and orders are chosen a priori. In summary, the general dual-band model can be divided into four different blocks, whose expressions for the lower frequency band are:

- Linear block $\left(p_{1}=p_{2}=0\right)$ :

$$
x_{1}\left(k-q_{0}\right) .
$$

- Intraband block $\left(p_{2}=0\right)$ :

$$
x_{1}\left(k-q_{0}\right)\left|x_{1}\left(k-q_{1}\right)\right|^{p_{1}} .
$$

- Crossband block $\left(p_{1}=0\right)$ :

$$
x_{1}\left(k-q_{0}\right)\left|x_{2}\left(k-q_{2}\right)\right|^{p_{2}} .
$$

- MP mixed-band block:

$$
x_{1}\left(k-q_{0}\right)\left|x_{1}\left(k-q_{0}\right)\right|^{p_{1}}\left|x_{2}\left(k-q_{0}\right)\right|^{p_{2}} .
$$

As shown above, it is common in literature that the mixedband block only contains a 2-D MP model, and not the leading or lagging terms. This dual-band model, which will be called from now on 2-D GMP, includes the most important aspects of the state-of-the-art models. However, pruning of some relevant regressors is identified and their incorporation to the model can improve its performance as it will be presented next.

\section{Proposed UpGRAdED MOdEL}

In GMP model, as shown in (1), the regressors with an even value of $p=2 r$ contain delayed versions of $|x(k)|^{2 r}$. For a dual-band signal

$$
x(k)=x_{1}(k) e^{-j \Omega k}+x_{2}(k) e^{j \Omega k},
$$

the signals $x_{1}(k)$ and $x_{2}(k)$ are centered in the first zones of the first band (B1) and second band (B2), i.e., in $-\Omega$ and $+\Omega$, respectively.

$$
\begin{aligned}
|x(k)|^{2 r}=\left(\left|x_{1}(k) e^{-j \Omega k}+x_{2}(k) e^{j \Omega k}\right|^{2}\right)^{r}= & \\
=\left[\left|x_{1}(k)\right|^{2}+\left|x_{2}(k)\right|^{2}+\right. & x_{1}(k) x_{2}^{*}(k) e^{-j 2 \Omega k}+ \\
& \left.+x_{1}^{*}(k) x_{2}(k) e^{j 2 \Omega k}\right]^{r} .
\end{aligned}
$$

In mathematics, the multinomial theorem describes how to expand a power of a sum in terms of powers of the terms in that sum. Its expression is

$$
\left(x_{1}+x_{2}+\cdots+x_{m}\right)^{r}=\sum_{s_{1}+s_{2}+\cdots+s_{m}=r} \frac{r !}{s_{1} ! s_{2} ! \cdots s_{m} !} \prod_{t=1}^{m} x_{t}^{s_{t}} \text {. }
$$

Using (10), (9) can be written as

$$
\begin{gathered}
|x(k)|^{2 r}=\sum_{s_{1}+s_{2}+s_{3}+s_{4}=r} \frac{r !}{s_{1} ! s_{2} ! s_{3} ! s_{4} !} \times \\
\times\left|x_{1}(k)\right|^{2 s_{1}}\left|x_{2}(k)\right|^{2 s_{2}} x_{1}^{s_{3}}(k) x_{2}^{*, s_{3}}(k) x_{1}^{*, s_{4}}(k) x_{2}^{s_{4}} e^{j 2\left(s_{4}-s_{3}\right) \Omega k} .
\end{gathered}
$$

It demonstrates responses in the even zones $0, \pm 2 \Omega, \pm 4 \Omega, \ldots$, and combined with (1) some of them produce two types of regressors in the first zone $\mathrm{B} 1$ :

- The first type of regressors is generated by the responses in the center zone $\left(s_{3}=s_{4}\right)$, that combine with $x_{1}(k)$ to produce

$$
x_{1}\left(k-q_{1}\right)\left|x_{1}\left(k-q_{1}-q_{2}\right)\right|^{2 s_{1}+2 s_{4}}\left|x_{2}\left(k-q_{1}-q_{2}\right)\right|^{2 s_{2}+2 s_{4}} .
$$

- The second type of regressors is generated by the responses in the second lower zone, centered at $-2 \Omega$ $\left(s_{4}-s_{3}=-1\right)$, that combine with $x_{2}(k)$ to produce

$$
\begin{aligned}
& x_{2}\left(k-q_{1}\right) x_{1}\left(k-q_{1}-q_{2}\right)\left|x_{1}\left(k-q_{1}-q_{2}\right)\right|^{2 s_{1}+2 s_{4}} \\
& \quad \times x_{2}^{*}\left(k-q_{1}-q_{2}\right)\left|x_{2}\left(k-q_{1}-q_{2}\right)\right|^{2 s_{2}+2 s_{4}} .
\end{aligned}
$$

The regressors of the first type can be customarily separated into the four blocks of the 2-D GMP model, although the mixed-band block is extended so that it has the same memory structure as the GMP model. The second type of regressors form a novel mixed-band block, given by (13).

Although this analysis has been made for even values of $p$, it can be extended to terms with an odd value of $p$ following a similar reasoning. Also, the expression for the response inside B2 is given with regressors obtained by swapping the signals $x_{1}(k)$ and $x_{2}(k)$ in the above equations.

In conclusion, the new upgraded model, 2-D U-GMP, can be summed up to be a GMP extension in the mixed-band model so that leading and lagging terms are minded, $\phi_{j, i}^{(1)}$, and also a new mixed-band block is included, $\phi_{j, i}^{(2)}$, which is obtained through the mathematical analysis expressed in this section. Its full expression for the first frequency band is

$$
\begin{aligned}
u_{1}(k) & =\sum_{t=1}^{2} \sum_{j} h_{t, j, 1} \phi_{j, 1}^{(t)}\left\{x_{1}(k), x_{2}(k)\right\}, \\
\phi_{j, 1}^{(1)}\left\{x_{1}(k), x_{2}(k)\right\} & =x_{1}\left(k-q_{0}\right)\left|x_{1}\left(k-q_{1}\right)\right|^{p_{1}}\left|x_{2}\left(k-q_{1}\right)\right|^{p_{2}}, \\
\phi_{j, 1}^{(2)}\left\{x_{1}(k), x_{2}(k)\right\} & =x_{2}\left(k-q_{0}\right) x_{1}\left(k-q_{1}\right)\left|x_{1}\left(k-q_{1}\right)\right|^{p_{1}} \\
& \times x_{2}^{*}\left(k-q_{1}\right)\left|x_{2}\left(k-q_{1}\right)\right|^{p_{2}} .
\end{aligned}
$$

The model expression for the second frequency band is akin to the previous equation, just swapping the subscripts of the signals. 


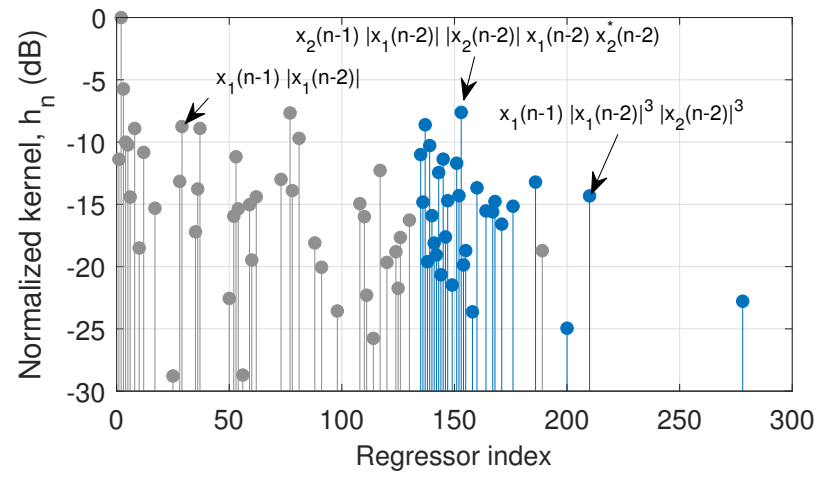

Fig. 1. Absolute value of selected Volterra coefficients normalized with respect to the highest one. Selected regressors from the new block of the 2-D U-GMP model are highlighted.

TABLE I

LINEARIZATION PERFORMANCE OF THE SYSTEM WITHOUT DPD AND THE LINEARIZED SIGNAL USING 2-D GMP DPD AND 2-D U-GMP DPD

\begin{tabular}{ccccc}
\hline \hline Model & $\begin{array}{c}\text { NMSE } \\
(\mathrm{dB})\end{array}$ & $\begin{array}{c}\mathrm{ACPR}_{1 \mathrm{~L} / 1 \mathrm{U}} \\
(\mathrm{dBc})\end{array}$ & $\begin{array}{c}\mathrm{ACPR}_{2 \mathrm{~L} / 2 \mathrm{U}} \\
(\mathrm{dBc})\end{array}$ & $\begin{array}{c}\mathrm{EVM}_{1 / 2} \\
(\%)\end{array}$ \\
\hline w/o DPD & -20.9 & $-35.3 /-34.2$ & $-37.0 /-39.4$ & $4.8 / 3.1$ \\
2-D GMP & -34.3 & $-43.4 /-44.7$ & $-42.5 /-45.4$ & $1.7 / 2.1$ \\
2-D U-GMP & -38.0 & $-45.5 /-45.3$ & $-45.6 /-47.5$ & $1.3 / 1.4$ \\
\hline \hline
\end{tabular}

\section{EXPERIMENTAL SETUP AND RESUlts}

A comparative assessment of the linearization performance when using two different DPD models was carried out. One is based on the standard 2-D GMP model and the other includes the new mixed-band terms proposed in this communication, or 2-D U-GMP model. The RF WebLab maintained by Chalmers University of Technology [15] was employed, whose measurement setup contains a Vector Signal Transceiver with $200 \mathrm{MHz}$ instantaneous bandwidth and center frequency of $2.14 \mathrm{GHz}$, a linear driver amplifier with a gain of $40 \mathrm{~dB}$, a GaN PA based on the Cree transistor CGH40006P, and a $30 \mathrm{~dB}$ attenuator.

The dual-band probing signal was composed by two orthogonal frequency division multiplexing (OFDM) signals according to the 5G-NR standard, each with $20 \mathrm{MHz}$ bandwidth and $30 \mathrm{kHz}$ separation between subcarriers, and both bands had a separation of $80 \mathrm{MHz}$. It is worth mentioning that the original PAPR of the combined signal was lowered to $10 \mathrm{~dB}$ using CFR techniques in order not to exceed the measurement setup restrictions [16]. The average output power was $27.7 \mathrm{dBm}$ and the normalized mean squared error (NMSE) between the input and output signals without predistortion presented a value of $-20.9 \mathrm{~dB}$. Both input and output signals were split into two single-band signals with a sampling frequency of $80 \mathrm{MHz}$, and then used to compute the DPDs.

The 2-D GMP model was configured with the parameters $\mathcal{K}_{a}=7, \mathcal{L}_{a}=3$ (only in linear block, $\mathcal{L}_{a}=5$ ), $\mathcal{K}_{b}=\mathcal{K}_{c}=5$, $\mathcal{L}_{b}=\mathcal{L}_{c}=3, \mathcal{M}_{b}=\mathcal{M}_{c}=2$, using the same notation as in (24) of [10]. Therefore, it had approximately 300 possible regressors in each frequency band. The same configuration was applied on the 2-D U-GMP and almost 500 possible regressors were obtained. This way, its memory depth and regressor order

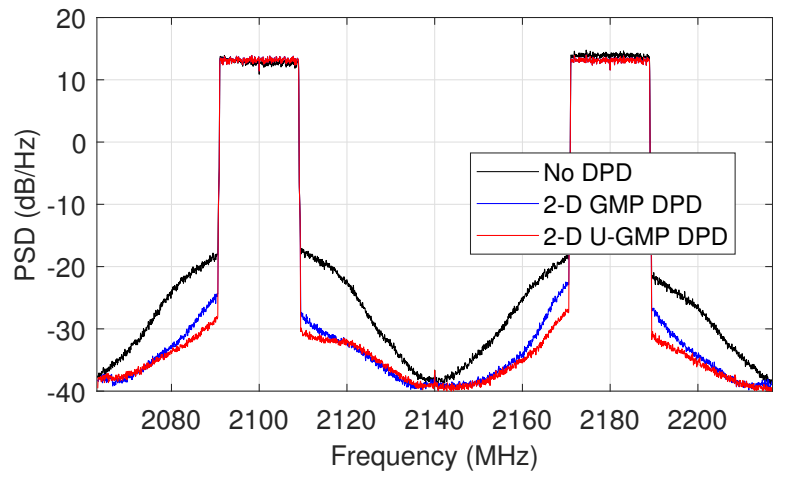

Fig. 2. Power spectral densities of the output signal without DPD, and of the linearized output signals for 2-D GMP DPD and 2-D U-GMP DPD.

had to be reduced so that the size of the regressor pool was similar and both models had the same complexity.

As overfitting may be a problem when the models have this high number of components, a pruning strategy has to be employed. Therefore, once all the regressors were built, DOMP algorithm [9] was executed to sort them by their importance, and then the Bayesian information criterion (BIC) was applied to attain the number of needed regressors and accomplish a good performance. For the 2-D GMP model, there were 42 and 39 selected regressors for each frequency band, but for the 2-D U-GMP model, 72 and 96 regressors were chosen. Fig. 1 shows the value of the normalized Volterra coefficients of the selected regressors for the lower band, where the regressors of the proposed upgrade are highlighted. It is remarkable the high number of selected regressors from the proposed extension, which therefore are not included in the original model: 4 from the memory expansion of the mixedband block of the 2-D GMP model and 26 from the new regressor type, $\phi_{j, i}^{(2)}$. An equivalent behavior is observed in the upper frequency band. In general, the proposed ensemble of selected regressors is richer, as more regressors are selected with the same initial number for both models.

After both DPDs were identified in an indirect learning architecture, the predistorted signals were calculated and used as inputs in two different measurements of the RF WebLab. The power spectral density (PSD) of both linearized output signals are depicted in Fig. 2, where the enhancement in linearization performance produced by the upgraded model can be observed. Linearization performances of both techniques are compared in Table I, where the subscripts 1 and 2 stand for the first and second band respectively, while $\mathrm{L}$ and $\mathrm{U}$ denote the lower or upper adjacent channel.

\section{CONCLUSion}

The 2-D U-GMP model is presented in this work as an upgrading of the 2-D GMP model. Theoretical analysis of its origin is treated, and an experimental linearization has been carried out in order to compare this extension to the original model. The attained linearization performance and the selected regressors by the DOMP algorithm conclude that this upgrading is beneficial for nonlinearity mitigation when dealing with dual-band signals. 


\section{REFERENCES}

[1] P. Roblin, S. K. Myoung, D. Chaillot, Y. G. Kim, A. Fathimulla, J. Strahler, and S. Bibyk, "Frequency-selective predistortion linearization of RF power amplifiers," IEEE Trans. Microw. Theory Techn., vol. 56, no. 1, pp. 65-76, Jan. 2008.

[2] S. A. Bassam, W. Chen, M. Helaoui, F. M. Ghannouchi, and Z. Feng, "Linearization of concurrent dual-band power amplifier based on 2DDPD technique," IEEE Microw. Wireless Compon. Lett., vol. 21, no. 12, pp. 685-687, Dec. 2011.

[3] S. A. Bassam, M. Helaoui, and F. M. Ghannouchi, "2-D digital predistortion (2-D-DPD) architecture for concurrent dual-band transmitters," IEEE Trans. Microw. Theory Techn., vol. 59, no. 10, pp. 2547-2553, Oct. 2011.

[4] Y. Liu, W. Chen, J. Zhou, B. Zhou, and F. M. Ghannouchi, "Digital predistortion for concurrent dual-band transmitters using 2-D modified memory polynomials," IEEE Trans. Microw. Theory Techn., vol. 61, no. 1, pp. 281-290, Jan. 2013.

[5] C. Wang, W. Zhu, and X.-W. Zhu, "A new form of polynomial model for concurrent dual-band digital predistortion," in 2015 IEEE Topical Conference on Power Amplifiers for Wireless and Radio Applications (PAWR). IEEE, Jan. 2015.

[6] L. Ding, Z. Yang, and H. Gandhi, "Concurrent dual-band digital predistortion," in 2012 IEEE/MTT-S International Microwave Symposium Digest (MTT). IEEE, Jun. 2012.

[7] F. Mkadem, A. Islam, and S. Boumaiza, "Multi-Band ComplexityReduced Generalized-Memory-Polynomial Power-Amplifier Digital Predistortion," IEEE Trans. Microw. Theory Techn., vol. 64, no. 6, pp. 17631774, Jun. 2016.

[8] H. Enzinger, K. Freiberger, and C. Vogel, "Competitive Linearity for Envelope Tracking: Dual-Band Crest Factor Reduction and 2D-VectorSwitched Digital Predistortion," IEEE Microw. Mag., vol. 19, no. 1, pp. 69-77, Jan. 2018.

[9] J. A. Becerra, M. J. Madero-Ayora, and C. Crespo-Cadenas, "Comparative analysis of greedy pursuits for the order reduction of wideband digital predistorters," IEEE Trans. Microw. Theory Techn., vol. 67, no. 9, pp. 3575-3585, Sep. 2019.

[10] D. R. Morgan, Z. Ma, J. Kim, M. G. Zierdt, and J. Pastalan, “A Generalized Memory Polynomial Model for Digital Predistortion of RF Power Amplifiers," IEEE Trans. Sig. Proc., vol. 54, no. 10, pp. 3852 3860, Oct. 2006.

[11] C. Quindroit, N. Naraharisetti, P. Roblin, S. Gheitanchi, V. Mauer, and M. Fitton, "Concurrent dual-band digital predistortion for power amplifier based on orthogonal polynomials," in 2013 IEEE MTT-S International Microwave Symposium Digest (MTT). IEEE, Jun. 2013.

[12] G. Yang, F. Liu, L. Li, H. Wang, C. Zhao, and Z. Wang, "2D orthogonal polynomials for concurrent dual-band digital predistortion," in 2013 IEEE MTT-S International Microwave Symposium Digest (MTT). IEEE, Jun. 2013.

[13] P. L. Gilabert, G. Montoro, D. Lopez, and J. A. Garcia, "3D Digital predistortion for dual-band envelope tracking power amplifiers," in 2013 Asia-Pacific Microwave Conference Proceedings (APMC). IEEE, Nov. 2013.

[14] Q. Lu, F. Meng, R. Zhang, J. Chen, and C. Yu, "Digital Predistortion for a Dual-Band Envelope-Tracking Power Amplifier with reduced bandwidth requirement for RF and envelope path," in 2017 Sixth AsiaPacific Conference on Antennas and Propagation (APCAP). IEEE, Oct. 2017.

[15] Chalmers University of Technology, "RF WebLab." [Online]. Available: http://dpdcompetition.com/rfweblab/

[16] W.-J. Kim, K.-J. Cho, S. P. Stapleton, and J.-H. Kim, "An efficient crest factor reduction technique for wideband applications," Analog Integrated Circuits and Signal Processing, vol. 51, no. 1, pp. 19-26, Apr. 2007. 